\title{
Red Blood Cell Exchange in children with Sickle Cell Disease in French Guiana
}

Authors: Narcisse ELENGA ${ }^{1,2}$ MD, PhD, Vincent VANTILCKE ${ }^{1}$ MD, Elise MARTIN ${ }^{2}$ MD, Emma CUADRO ${ }^{2}$ MD, Mickael PARISOT ${ }^{1}$ MD, Françoise MAIRE ${ }^{3}$ MD, Pierre SELLES $^{3}$ MD, Thierry BASSET ${ }^{2}$ MD (in memoriam).

1Sickle cell disease centre, Andrée Rosemon Regional Hospital, Rue des flamboyants, BP 6006, 97306 Cayenne Cedex, French Guiana

2Pediatric Medicine and Surgery, Andrée Rosemon Regional Hospital, Rue des flamboyants, BP 6006, 97306 Cayenne Cedex, French Guiana

3Etablissement Français du Sang, Andrée Rosemon , Rue des flamboyants, BP 6006, 97306 Cayenne Cedex, French Guiana

\section{Running title: Transfusion Exchange in Sickle Cell Disease}

\section{Corresponding author:}

Narcisse ELENGA, MD, PhD

Pediatric Medicine and Surgery, Cayenne Hospital, Rue des flamboyants, BP 6006, 97306 Cayenne Cedex, French Guiana

Tel. $(+594) 694978048$ Fax (+594) $0594397737 / 7735$

Email: elengafr@yahoo.fr

\begin{abstract}
The aim of our study was to describe our experience using a Spectra Optia® automated apheresis system in children with sickle cell disease (SCD). We used automated red blood cell exchange (RCE) to treat acute and chronic complications in 75 children with SCD who
\end{abstract}


had a median age of 10 years [7-13]. We analysed 649 exchange sessions. Peripheral venous access was limited in a number of the children, thus requiring a femoral central double-lumen venous catheter (CVC). We recommend the use of heparin locking, with 500 units in each lumen of a CVC. This method was well tolerated, with few complications during the procedures. For preoperative prevention, all of the patients had achieved a post-RCE HbS level of $<30 \%$ since this is a mandatory condition imposed by the anaesthesiologist. With a post-RCE $\mathrm{Hb}$ level of approximately $10-11 \mathrm{~g} / \mathrm{dL}$, a blood exchange volume of $\geq 32 \mathrm{~mL} / \mathrm{kg}$, and an interval between each RCE procedure of $\leq 30$ days, it was able to maintain the residual $\mathrm{HbS}$ level below 30\%. Despite a target pre-exchange $\mathrm{HbS}$ level of $47 \%$, we did not encounter a single stroke recurrence. Erythrocytapheresis is useful and safe for children with SCD.

Keywords: Erythrocytapheresis; red blood cell exchange; sickle cell disease; target $\mathrm{HbS}$ level; heparin locking

\section{Introduction}

Sickle cell disease (SCD) refers to a group of autosomal recessive genetic disorders characterized by the synthesis of abnormal haemoglobin: sickle haemoglobin S. This is the result of the substitution of a single amino acid $(\mathrm{Glu} \rightarrow \mathrm{Val})$ at the sixth position of the $\beta$-chain of normal haemoglobin $(\mathrm{HbA})$ [1, 2 ]. This single point mutation leads to polymerization of $\mathrm{HbS}$ and red cell sickling under deoxygenated conditions. Homozygous SS is generally considered to be the most severe form of SCD. Compound heterozygotes, in whom $\mathrm{HbS}$ is combined with a different mutation in the second $\beta$-globin gene, such as $\mathrm{HbC}, \mathrm{D}, \mathrm{OArab}$, or $\beta$-thalassemia can also be affected, with variable genotypes. SCD can result in severe complications including chronic pain, end-organ dysfunction, stroke, life-long suffering, poor quality of life, and early mortality. Treatments include medications to reduce pain and to 
prevent complications (e.g., hydroxyurea), and blood transfusions, as well as a bone marrow transplant in patients who have a related donor $[3,4]$.

Red blood cell (RBC) transfusions are often used to treat acute complications of SCD. The purpose of RBC transfusion is to increase oxygen distribution to the tissues and/or to replace the rigid sickle-shaped RBCs with healthy deformable RBCs [5 ]. Transfusion can also be part of a regular long-term transfusion program to prevent SCD complications. When the goal is reduction of the $\mathrm{HbS}$ level, exchange transfusion is the therapy of choice [6, 7 ] as it allows for better control of the blood volume and viscosity, in addition to decreasing the risk of iron overload [8 ]. A red blood cell (RBE) exchange can be accomplished manually by bleeding and transfusion or through the use of an apheresis device that separates the plasma from the RBCs, removes and replaces the patient's red blood cells, and then returns the plasma to the body [9-12 ]. The aim of our study was to describe our experience using a Spectra Optia® automated apheresis system in children with SCD [13, 14].

\section{Materials and Methods}

\section{Study site}

Cayenne Hospital is a 510-bed general medical centre that is a referral and teaching hospital, and it runs the only sickle cell centre for children and adults (created in September 2014) in the country. The study population was part of a pre-existing cohort, Improving the quality of management of SCD in French Guiana: "Epidemiology of predictive factors of acute clinical events," that enrolled approximately1,000 patients.

\section{Study design}

Since the implementation of the Spectra Optia ${ }^{\circledR}$ system in our department in April of 2012, we have exclusively used the Spectra Optia ${ }^{\circledR}$ Apheresis System for RBC exchange. The Spectra Optia ${ }^{\circledR}$ automated apheresis system is manufactured and supplied by Terumo Medical 
Corporation. It has several applications relating to the in-situ separation of blood components, including automated RBE exchange and depletion exchange in adults or children with sickle cell disease. Since 2017, for patients with abnormal transcranial Doppler (TCD) velocities, we provide a transfusion exchange program for a year before switching to hydroxyurea (HU) [15, $16]$.

\section{Exchange transfusion procedure}

The vascular access consisted of either peripheral vein or femoral central double-lumen venous catheterisation. Using each patient's height, weight, gender, pre-RCE Hct, pre-RCE $\mathrm{HbS}$, post-RCE Hct goal, and post-RCE HbS goal, the volume of RBCs to be used in each procedure was calculated automatically when their weight was $>25 \mathrm{~kg}$. For the patients weighing less than $25 \mathrm{~kg}$, the volume of RBCs in millilitres was calculated by the formula Weight x70. The RCEs were performed by a nurse, with constant medical supervision ,as previously described $[15,16]$. Given the frequent presence of clots in the lumen of the central catheter, which makes the procedure difficult; we decided to administer heparin-lock solution, with 500 units in each lumen of a double-lumen central venous catheter.

Since April 2012, we have prospectively recorded all of the information regarding exchange transfusions in adults and children with SCD at our centre in an Excel database. All of the patients underwent a complete blood count, determination of the levels of ferritin, LDH, and serum electrolytes, as well as haemoglobin electrophoresis, in the $24-72$ hours prior to each RCE procedure. Our SCD population exclusively received transfusions with blood systematically matched for ABO, Rh, C, c, E, e, and Kel antigens. After each RCE procedure, the following parameters before and after the RCE were recorded: RCE, $\mathrm{Hb}, \mathrm{HbS}$, ferritin, and LDH levels. The other parameters were the indication for RCE, the intravenous access, 
the RCE duration, the quantity of blood exchanged in $\mathrm{ml} / \mathrm{kg}$, the interval between two RCE procedures, adverse events, and complications.

\section{Ethical and regulatory aspects}

The parents or authorized representatives of the patients provided written and informed consent to participate in this research. The study cohort was presented to the Cayenne General Hospital Ethical Committee (Number 1-2017-V2) and the database was declared to the French Data Protection Authority (Number 3Yj157849 3\#).

\section{Statistical analyses}

The data were analysed using STATA 15.0 (Stata Corp LP, College Station, TX, USA) software. The results are expressed as medians \pm the standard deviation. Fisher's exact test was used to study the categorical variables while the Kruskal-Wallis test was used to study the non-Gaussian variables. The factors associated with the outcomes were analysed by unconditional multiple logistic regression. For all of the tests, a p-value of 0.05 or less was considered statistically significant. We used a stepwise univariate model to calculate the best blood exchange quantity, the best post-Hb level, and the best interval between RCE procedures.

\section{Results}

Seventy-five children, with a median age of 10 years [7-13], were enrolled. There were 30 females and 45 males, of whom 64 had the SS genotype (85\%), 8 were SC (11\%,) and 3 were S- $\beta$ Thalassemia (4\%). The transfusions were carried out manually 23 times $(3 \%)$ and by automated RCE 626 times (97\%). Figure 1 shows the distribution of the RCE procedures performed at our centre since 2012 .

\section{Exchange transfusion indications}


The preoperative prevention was the first indication, followed by acute chest syndrome (Table 1). For the preoperative prevention, all of the patients had achieved a post-RCE HbS level of $<30 \%$, as this is a mandatory condition imposed by the anaesthesiologist.

\section{Side effects and complications}

The side effects and complications are summarized in Table 2.

\section{Intravenous access}

A femoral central catheter was used 353 times (54\%) and the peripheral vein 296 times (46\%) ( Figure 2). None of the patients had an arteriovenous fistula. We frequently used Doppler ultrasound to find the vein (Figure 3).

\section{Patients with chronic transfusion}

Between 2012 and 2019, 17 children (12 boys and 5 girls), with a median age of 8 [7-10] years were placed on chronic transfusion for stroke prevention at our centre. Of these, 16 had homozygous SCD and one had sickle $\beta^{0}$-thalassaemia. Eight had a history of stroke and nine an abnormal transcranial Doppler (TCD)velocity. One patient developed red blood cell alloantibodies. Of the 17 patients, 4 required iron chelation. For $24 \%$ of the patients, the $\mathrm{HbS}$ level was below 30\%. The target median $\mathrm{HbS}$ level was $47 \%$ [30-53]. None of the patients experienced a stroke recurrence. In order to understand why there were relatively few patients with a residual $\mathrm{HbS}$ level below $30 \%$, we performed a logistic regression in order to determine the optimal target post-RCE levels for haemoglobin, the haematocrit, and the interval between two RCE procedures to reduce the HbS level below 30\%. Based on the results of stepwise logistic regression ( Table 3), we came to the conclusion that the apheresis machine should be programmed with the aim of achieving a post-exchange $\mathrm{Hb}$ level of 
approximately10-11 g/dL (or a post-exchange haematocrit at $\sim 30 \%)(\mathrm{p}<0.001)$. The best total exchange volume to maintain a residual $\mathrm{HbS}$ level of $<30 \%$ was $>32 \mathrm{~mL} / \mathrm{kg}$ of body weight $(p=0.006)$. The best interval to maintain residual $\mathrm{HbS}$ level of $<30 \%$ between each RCE procedure was $\leq 30$ days $(\mathrm{p}<0.001)$. All of the patients undergoing chronic transfusion were given extensively matched units of packed red blood cells. One patient developed red blood cell alloantibodies. None of the patients underwent seroconversion for hepatitis B.

\section{Discussion}

Controlled studies have shown that TCD is effective at identifying children at high risk for stroke $[17,18]$. The Stroke Prevention Trial in SCD, by Adams et al., found a 92\% reduction in the annual incidence of primary stroke in children with an abnormal TCD velocity [19]. Currently, the standard care to reduce the incidence of primary and secondary stroke is discontinuing chronic transfusion allowing the target $\mathrm{HbS}$ level to be maintained at $<30 \%$. Although the methods of transfusion in these trials included simple transfusion and RCE, in our centre, we opted for erythrocytapheresis, as apheresis machines have been available in our hospital since 2012. In addition, erythrocytapheresis reduces iron overload and it allows for a longer interval between the RCE procedures [20]. The main problem, however, with this procedure is the significantly increased blood requirements [21]. In French Guiana, uncertainty regarding the safety of blood products led to the cessation of blood donations in April 2005[22]. Approximately two-thirds of the supply of packed RBC in French Guiana comes from mainland France and one-third comes from the Guadeloupian French Blood Establishment. Thus, erythrocytapheresis could have been affected at our centre. Fortunately, we have not experienced any problems to date with the blood supply. Despite the disparity between the donors and the recipients, only one patient in our study developed new RBC antibodies. However, none of our patients experienced seroconversion for hepatitis B, hepatitis C, or HIV. These findings support the feasibility of chronic transfusion in patients 
with SCD at our centre. In accordance with the literature, long-term transfusion therapy has been associated with complications, such as transfusional iron overload, transfusion reactions, thromboembolic complications, and allergies [23]. These side effects were minimal, however, because we exclusively used the automated apheresis system. In addition, the use of heparin locking allowed us to minimize the risk of thrombosis. We consequently highly recommend the use of this procedure. We did not compare the overall costs between manual exchange transfusion and erythrocytapheresis. Several authors have claimed that the cost of erythrocytapheresis is not higher than that of manual exchange transfusions [24-26]. We set a target $\mathrm{HbS}$ level of $30 \%$. In our study, only $24 \%$ of the patients achieved an $\mathrm{HbS}$ level below $30 \%$ after chronic transfusion, despite the use of erythrocytapheresis. This could be due to a lack of homogeneity in our practices. However, higher $\mathrm{HbS}$ levels prior to the next exchange transfusion have frequently been reported [27-29], thereby underscoring the difficulty with complying with the recommendations. Moreover, the statistical analysis showed that with a post-exchange $\mathrm{Hb}$ level of approximately $10-11 \mathrm{~g} / \mathrm{dL}$, a blood exchange volume of $\geq 32$ $\mathrm{mL} / \mathrm{kg}$, and an interval of $\leq 30$ days between each RCE procedure, the residual HbS level can be maintained below 30\%. It is often difficult though to attain this level. However, the optimal pre-exchange $\mathrm{Hb}$ level for stroke prevention has not been clearly defined. Cohen et al. have confirmed that a target pretransfusion $\mathrm{HbS}$ level of $50 \%$ imparts a high level of protection against recurrent stroke in SCD [30]. Moreover, in the SWiTCH study in the United States, pretransfusion $\mathrm{HbS}$ levels ranged between 22 and51\% in the various centres [31]. Some studies have reported a target $\mathrm{HbS}$ level of $>30 \%$ during stroke recurrence [32, 33]. Despite a target $\mathrm{HbS}$ level of $47 \%$, we did not encounter a single stroke recurrence.

Although chronic transfusion is presently the only proven therapy for primary or secondary stroke prevention in sickle cell children, recent studies have demonstrated the efficacy of HU in maintaining normal TCD velocities after at least 1 year of chronic blood transfusion [34]. 
These studies are promising, as the duration of chronic transfusions is unknown when the child lacks a compatible HLA donor for a hematopoietic stem cell transplantation. Indeed, as in the case with other therapeutic aphereses, good vascular access is required, although this can often be difficult to obtain in paediatric sickle cell patients requiring a chronic exchange transfusion program. Whenever possible, peripheral access is ideal for RBCs because it is safer. With patients for whom peripheral venous access is impossible, a double-lumen femoral catheter (used for very short-term access) with a high flow is needed, and this can be associated with infections, thrombosis, and difficulties with blood withdrawal [35-37]. Arteriovenous fistulas have been suggested, ${ }^{37,38}$ but there has been a paucity of data to date in the literature regarding their use in children with $\operatorname{SCD}[37,39]$.

\section{Conclusion}

Erythrocytapheresis is useful and safe for patients with SCD who require a chronic exchange transfusion program. However, the optimal pretransfusion $\mathrm{HbS}$ level for stroke prevention remains unclear. Despite a target $\mathrm{HbS}$ level of $47 \%$, we did not encounter a single stroke recurrence. This procedure is less time consuming for nurses and patients and it improves the iron overload. It can also be performed successfully in children without having to resort to using a central line.

\section{Acknowledgements}

The authors would like to thank Pr Mathieu NACHER from the INSERM U1424 of Cayenne Hospital, Rue des Flamboyants, BP 6006, 97306 Cayenne Cedex, French Guiana, for his advice and corrections. 


\section{Authorship contributions}

NE analyzed the data and drafted the manuscript, VV, EM, EC, MP, PS and FM collected the data, provided necessary logistic support and have read the manuscript. NE and FM provided critical comments on the manuscript. I confirm that all authors read and approved the final manuscript.

\section{Disclosure of conflicts of interest}

The authors declare that they have no competing interests

\section{Funding and resources}

There is no fund related to this study.

\section{References}

1-Azar S, Wong TE. Sickle Cell Disease: A Brief Update. Med Clin North Am. 2017; 101(2): 375-393.

2- Patrick T. McGann, Alecia C. Nero, Russell E. Ware. Current Management of Sickle Cell Anemia Cold Spring Harb Perspect Med. 2013; 3(8): a011817.

3- Russo G, De Franceschi L, Colombatti R, et al. Current challenges in the management of patients with sickle cell disease - A report of the Italian experience. Orphanet J Rare Dis. 2019; $14(1): 120$. 
4- Gluckman E, Cappelli B, Bernaudin F, et al. Sickle cell disease: an international survey of results of HLA-identical sibling hematopoietic stem cell transplantation. Blood . 2017; 129(11): 1548-1556.

5- Merlin E, Hequet O, Kanold J. Red blood cell exchange in children and adolescents with sickle cell disease. Transfus Apher Sci. 2019; 58(2):136-141.

6- Fort R. Recommendations for the use of red blood cell exchange in sickle cell disease. Transfus Apher Sci. 2019;58(2):128-131..

7- Thibodeaux SR, Tanhehco YC, Irwin L, et al. More efficient exchange of sickle red blood cells can be achieved by exchanging the densest red blood cells: An ex vivo proof of concept study. TransfusApher Sci. 2019;58(1):100-106.

8- Dedeken L, Lê PQ, Rozen L, et al. Automated RBC exchange compared to manual exchange transfusion for children with sickle cell disease is cost-effective and reduces iron overload. Transfusion. 2018; 58(6):1356-1362.

9- Driss F, Hequet O. Red blood cell exchange techniques and methods. Transfus Apher Sci. 2019;58 (2):132-135.

10- Raba M. Selecting red blood cell units to perform RBCX in patients with sickle cell disease. Transfus Apher Sci. 2019; 58(2):142-146.

11- Mehra RA, Gupta SA, Borkar DB. Manual red cell exchange transfusion to avert sickle cell related complications. Asian J Transfus Sci. 2018;12(2):157-159.

12- Biller E, Zhao Y, Berg Met al. Red blood cell exchange in patients with sickle cell disease-indications and management: a review and consensus report by the therapeutic apheresis subsection of the AABB. Transfusion. 2018; 58(8):1965-1972. 
13- Choi SJ, Cho H, Eom KS, et al. Effective sickle hemoglobin reduction by automated red cell exchange using Spectra Optia in three Emirati patients with sickle cell disease before allogeneic hematopoietic stem cell transplantation. Blood Res. 2018;53(4):325-329.

14-Willits I, Cole H, Jones R, et al. Spectra Optia(®) for Automated Red Blood Cell Exchange in Patients with Sickle Cell Disease: A NICE Medical Technology Guidance. Appl Health Econ Health Policy. 2017;15(4):455-468..

15- National Institute for Health and Care Excellence. Spectra Optia for automated red blood cell exchange in patients with sickle cell disease. 2016. Available from: https://www.nice.org.uk/guidance/indevelopment/gid-mt271. Accessed 8 Apr 2016.

16-Cedar Healthcare Technology Research Centre. Spectra Optia apheresis system for automated red blood cell exchange in patients with sickle cell disease. 2015. Available from: https://www.nice.org.uk/guidance/MTG28/documents/submission-of-evidence-by-sponsor-4. Accessed 13 Apr 2016.

17- Adams R. J, McKie VC, Nichols F, et al. The use of transcranial ultrasonography to predict stroke in sickle cell disease. N Engl J Med. 1992; 326: 605-610

18- Adams RJ., McKie VC., Carl EM, et al. Long-term stroke risk in children with sickle cell disease screened with transcranial Doppler. Ann Neurol 1977; 42: 699-704

19- Adams RJ., McKie VC, Hsu L, Bet al. Prevention of a first stroke by transfusions in children with sickle cell anemia and abnormal results on transcranial Doppler ultrasonography. N Engl J Med. 1998; 339: 5-11

20-Dedeken L, Lê PQ, Rozen L, et al. Automated RBC exchange compared to manual exchange transfusion for children with sickle cell disease is cost-effective and reduces iron overload. Transfusion. 2018 ;58 (6):1356-1362 
21- Howard J. Sickle cell disease: when and how to transfuse. Hematology Am Soc HematolEduc Program. 2016; 2016(1):625-631.

22- Aznar C, La Ruche G, Laventure S, et al. Seroprevalence of Trypanosoma cruzi infection in French Guiana. Mem Inst Oswaldo Cruz. 2004; 99(8):805-8

23- Estcourt LJ, Fortin PM, Hopewell S, et al. Blood transfusion for preventing primary and secondary stroke in people with sickle cell disease. Cochrane Database Syst Rev. 2017;1:CD003146.

25- Koehl A, Sommet J, Holvoet L, et al. Comparison of automated erythrocytapheresis versus manual exchange transfusion to treat cerebral macrovasculopathy in sickle cell anemia. Transfusion. 2016; 56: 1121- 8 .

26- Masera N, Tavecchia L, Pozzi L, et al. Periodic erythroexchange is an effective strategy for high risk paediatric patients with sickle-cell disease. Transfus Apher Sci. 2007; 37: 241 - 7.

27- Duclos C, Merlin E, Paillard C, et al. Long-term red blood cell exchange in children with sickle cell disease: manual or automatic? Transfus Apher Sci .2013; 48: 219- 22.

28-Kuo KH, Ward R, Kaya B, et al. A comparison of chronic manual and automated red blood cell exchange transfusion in sickle cell disease patients. Br J Hematol. 2015; 170: 4258.

29- Filhon B, Dumesnil C, Holtermann C, et al. Advantages and difficulties of an erythrocytapheresis program for sickle-cell patients: a pediatric experience. Arch Pediatr. 2012; 19: 572- 8.

30- Cohen AR, Martin MB, Silber JH, Kim HC, Ohene-Frempong K, Schwartz E. A modified transfusion program for prevention of stroke in sickle cell disease. Blood. 1992; 79: 1657-61. 
31- Aygun B, McMurray MA, Schultz WH, et al. Chronic transfusion practice for children with sickle cell anaemia and stroke. Br J Haematol. 2009;145:524-8

32-Pegelow CH, Adams RJ, McKie V, et al. Risk of recurrent stroke in patients with sickle cell disease treated with erythrocyte transfusions. J Pediatr. 1995;126:896-9

33-Scothorn DJ, Price C, Schwartz D, et al. Risk of recurrent stroke in children with sickle cell disease receiving blood transfusion for at least five years after initial stroke. J Pediatr. 2002;140:348-54.

34- Ware RE, Davis BR, Schultz WH, et al. Hydroxycarbamide versus chronic transfusion for maintenance of transcranial doppler flow velocities in children with sickle cell anaemia-TCD With Transfusions Changing to Hydroxyurea (TWiTCH): a multicentre, open-label, phase 3, non-inferiority trial. Lancet. 2016; 387 : 661-67.

35- Michon B, Moghrabi A, Winikoff R, et al. Complications of apheresis in children. Transfusion. 2007; 47: 1837- 42

36-Kara A, Turgut S, Cagli A, et al. Complications of therapeutic apheresis in children. Transfus Apheresis Sci. 2013; 48: 375- 6.

37- Delville M, Manceau S, Ait Abdallah N, et al. Arterio-venous fistula for automated red blood cells exchange in patients with sickle cell disease: Complications and outcomes. Am J Hematol. 2017;92(2):136-140.

38- Sarode R, Ballas SK, Garcia A, et al. Red blood cell exchange: 2015 American Society for Apheresis consensus conference on the management of patients with sickle cell disease. $J$ Clin Apher. 2017;32(5):342-367. 
39- Hartwig D, Schlager F, Bucsky P, et al. Successful long-term erythrocytapheresis therapy in a patient with symptomatic sickle-cell disease

using an arterio-venous fistula. Transfus Med. 2002;12:75-77. 